\title{
Pure shift NMR: Application of 1D PSYCHE and 1D TOCSY-PSYCHE Techniques for Directly Analyzing the Mixtures from Biomass-derived Platform Compound Hydrogenation/hydrogenolysis
}

Qi Zhao ${ }^{\dagger+}$, Hui Ma ${ }^{\dagger \ddagger}$, Christian M. Pedersen ${ }^{\S}$, Mengyu Dou ${ }^{\dagger}$, Yan Qiao ${ }^{\dagger *}$, Xianglin $\mathrm{Hou}^{\dagger+}$, Yongqin $\mathrm{Qi}^{\dagger}$, and Yingxiong Wang*广‡

† Shanxi Engineering Research Center of Biorefinery, Institute of Coal Chemistry, Chinese Academy of Sciences, 27 South Taoyuan Road, Taiyuan 030001, PR China

$\$$ Center of Materials Science and Optoelectronics Engineering, University of Chinese Academy of Sciences, Beijing 100049, PR China

$\S$ Department of Chemistry, University of Copenhagen, Universitetsparken 5, DK-2100, Copenhagen, Denmark

Corresponding Author E-mail: wangyx@sxicc.ac.cn

Supplementary Information Contents:

Number of pages: 7

Number of diagram: 1

Number of Figures: 9 


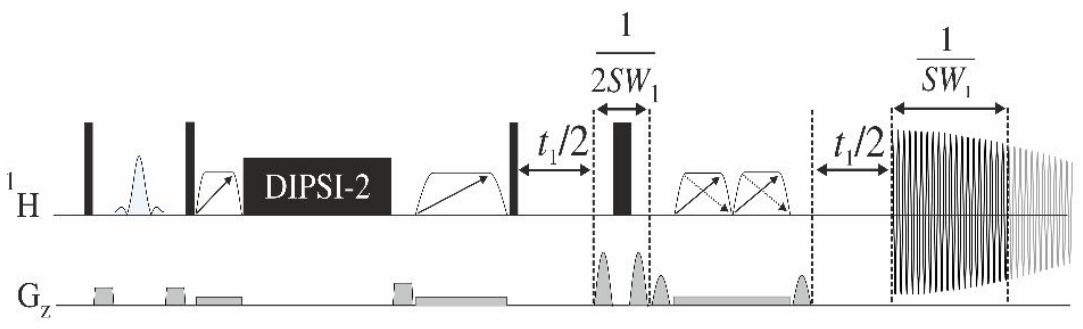

Figure S1. Pulse sequence of the 1D TOCSY-PSYCHE experiment. The hard $90^{\circ}$ and $180^{\circ}$ pulses are presented by narrow and wide rectangles, respectively. There two trapezoids with crossdiagonal arrows, which represent the low-power chirp pulses of flip angle $\beta\left(20^{\circ}\right)$. Trapezoids with one arrow are low power $180^{\circ}$ chirp pulses suppressing zero quantum coherences. ${ }^{1}$

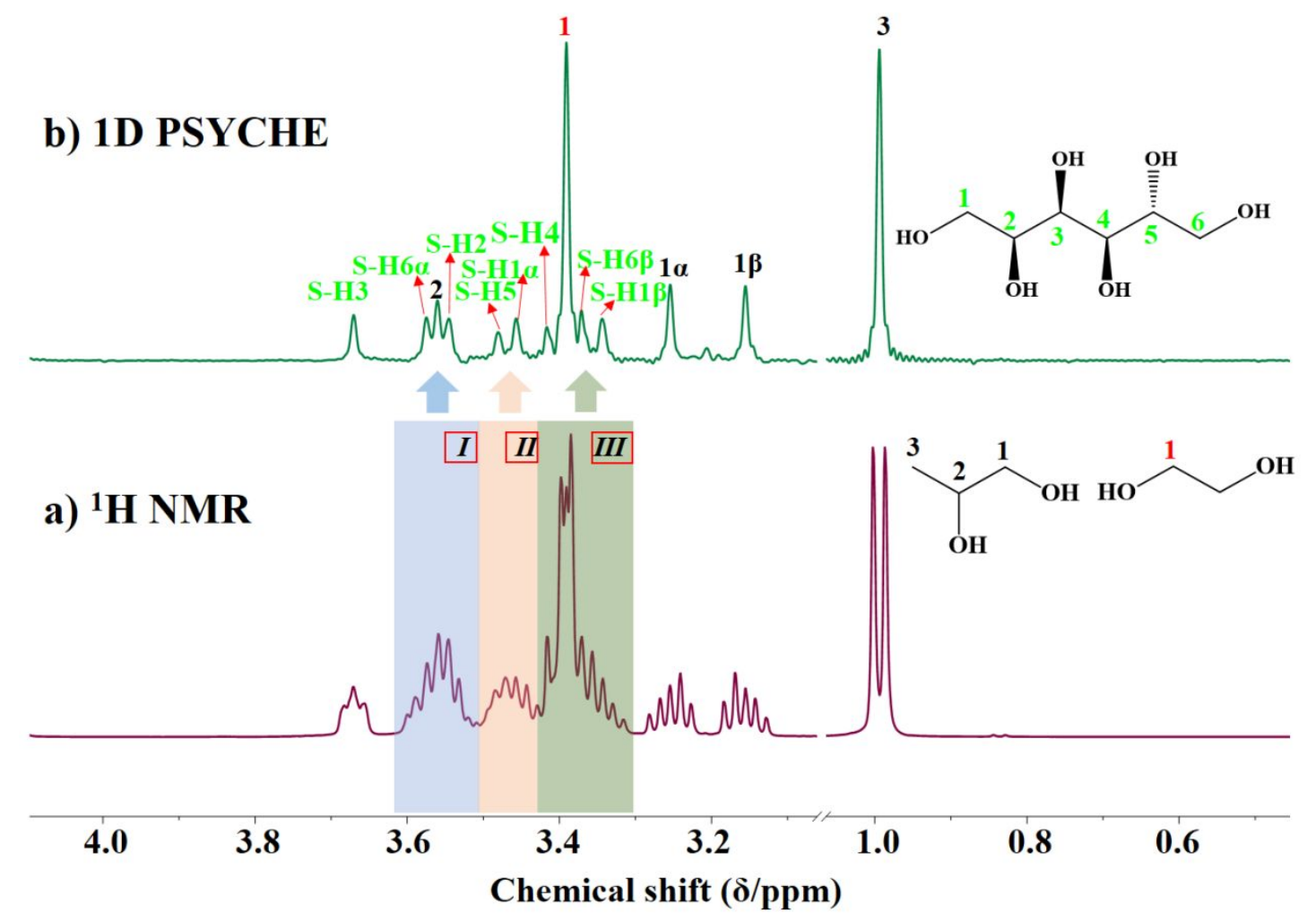

Figure S2. a) Conventional ${ }^{1} \mathrm{H}$ NMR spectrum b) 1D PSYCHE ${ }^{1} \mathrm{H}$ NMR spectrum of model mixture of sample 1 (sorbitol, 1,2-PG and EG) in DMSO- $d_{6}$ at $298 \mathrm{~K}$. The letter $\mathrm{S}$ stands for sorbitol. These signals can be assigned as follows: sorbitol (S-H3 $3.67 \mathrm{ppm}$, S-H6 3.57 ppm, S-H2 3.54 ppm, S-H5 3.48 ppm, S-H1 3.45 ppm, S-H4 3.42 ppm,

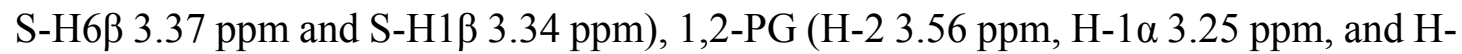
$1 \beta 3.16 \mathrm{ppm})$ and $\mathrm{EG}(\mathrm{H}-13.39 \mathrm{ppm})$. 

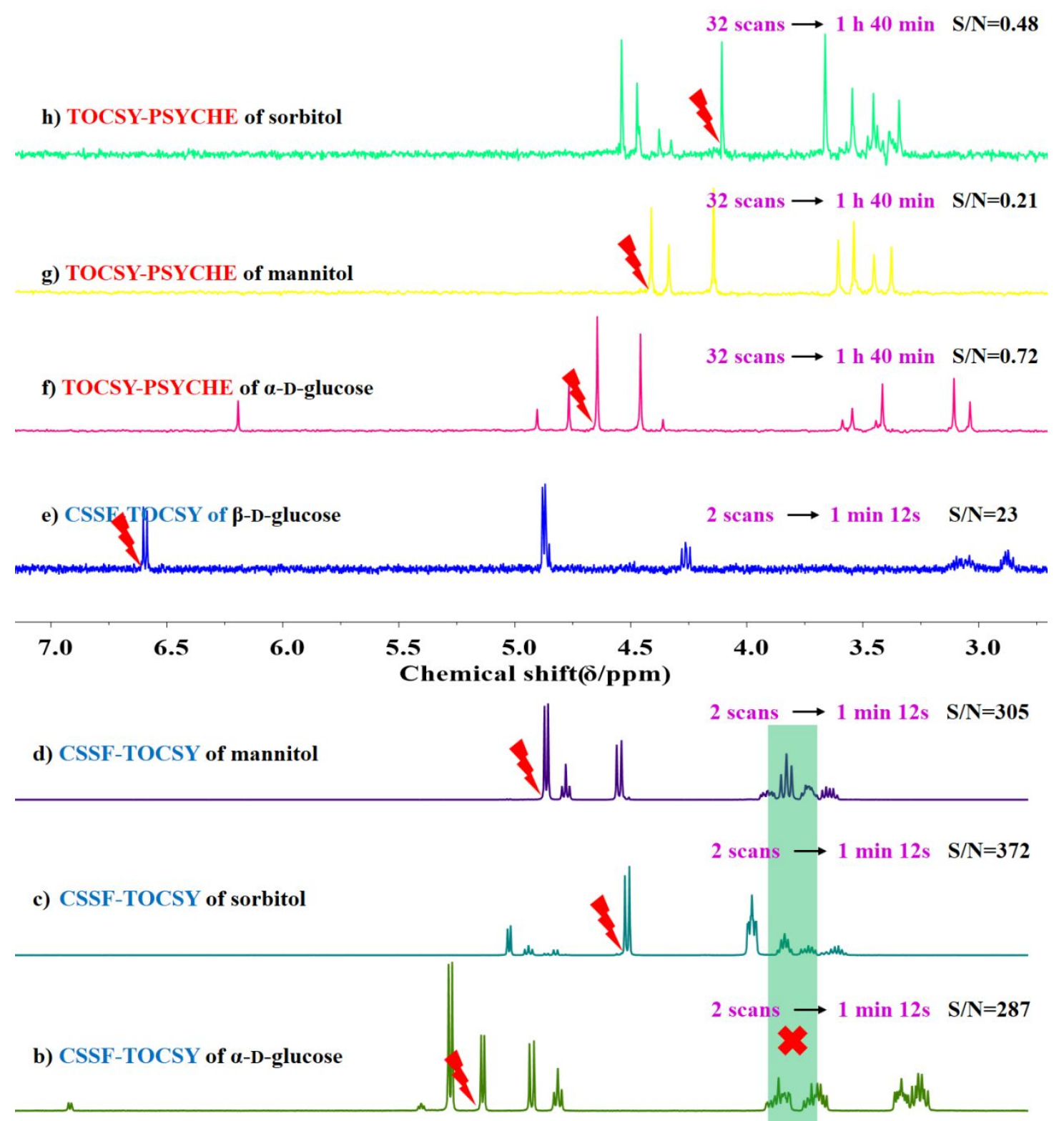

a) ${ }^{1} \mathrm{H}$ NMR

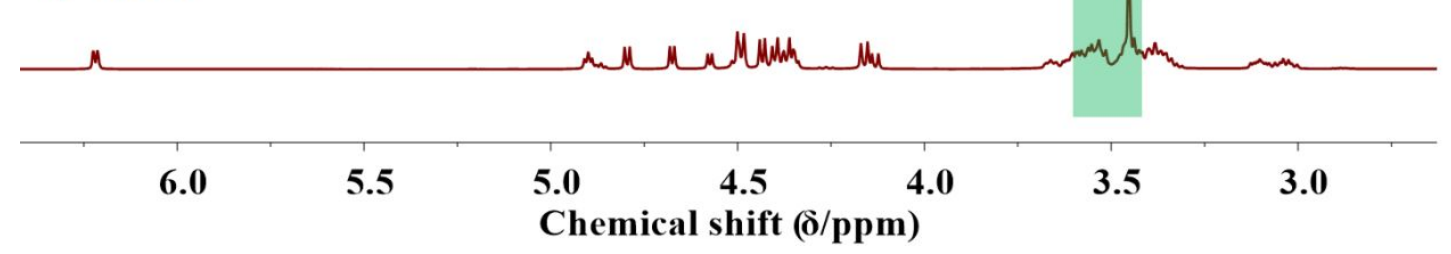

Figure S3. a) Conventional ${ }^{1} \mathrm{H}$ NMR spectrum of D-glucose hydrogenation reaction mixture (sample 2 including $\alpha$-D-glucose, sorbitol, mannitol and $\beta$-D-glucose), b-e) 1D CSSF-TOCSY spectra (selective excitation frequencies at 4.67, 4.13, 4.43 and 6.59 ppm, respectively) and f-h) 1D TOCSY-PSYCHE spectra of $\alpha$-D-glucose, mannitol and sorbitol (selective excitation frequencies at $4.67,4.43$ and 4.13 ppm, respectively) in 
DMSO- $d_{6}$ at $298 \mathrm{~K}$.

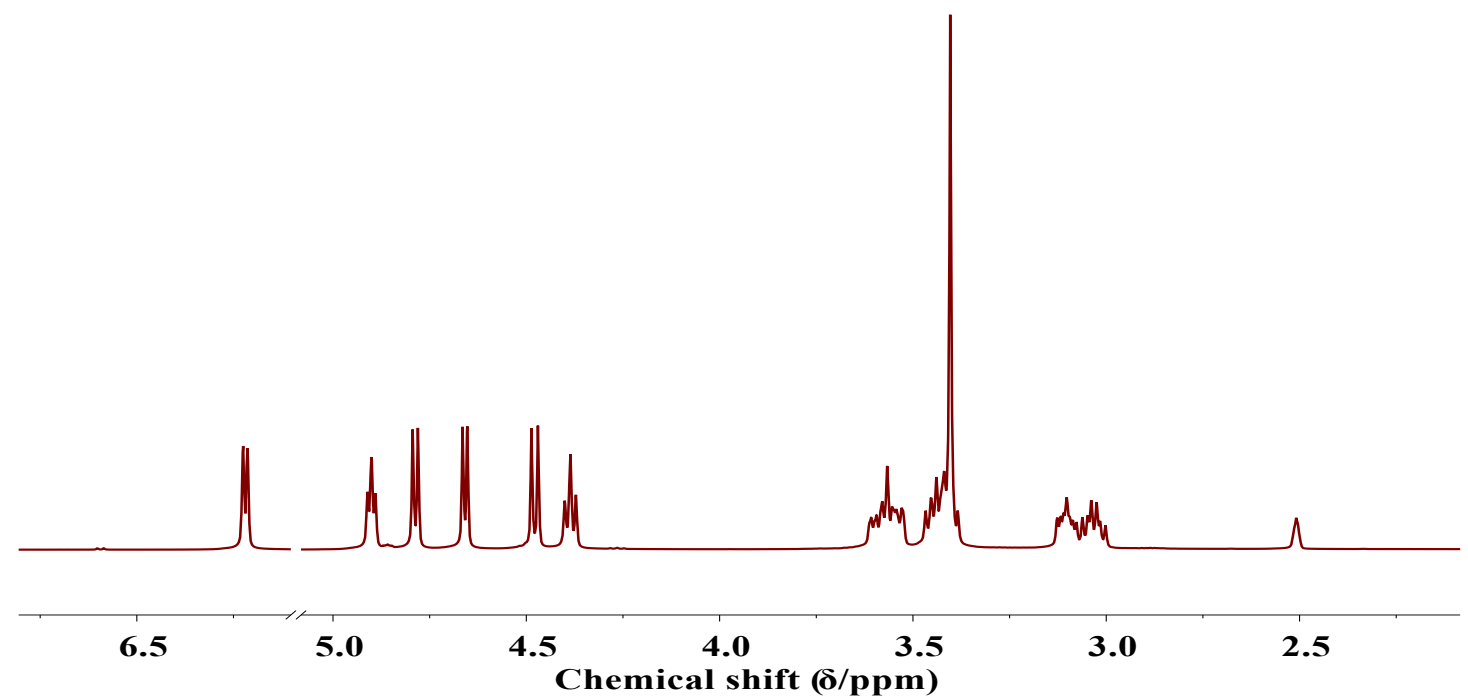

Figure S4. Conventional ${ }^{1} \mathrm{H}$ NMR spectrum of pure D- $\alpha$-glucose in DMSO- $d_{6}$ at 298 K.

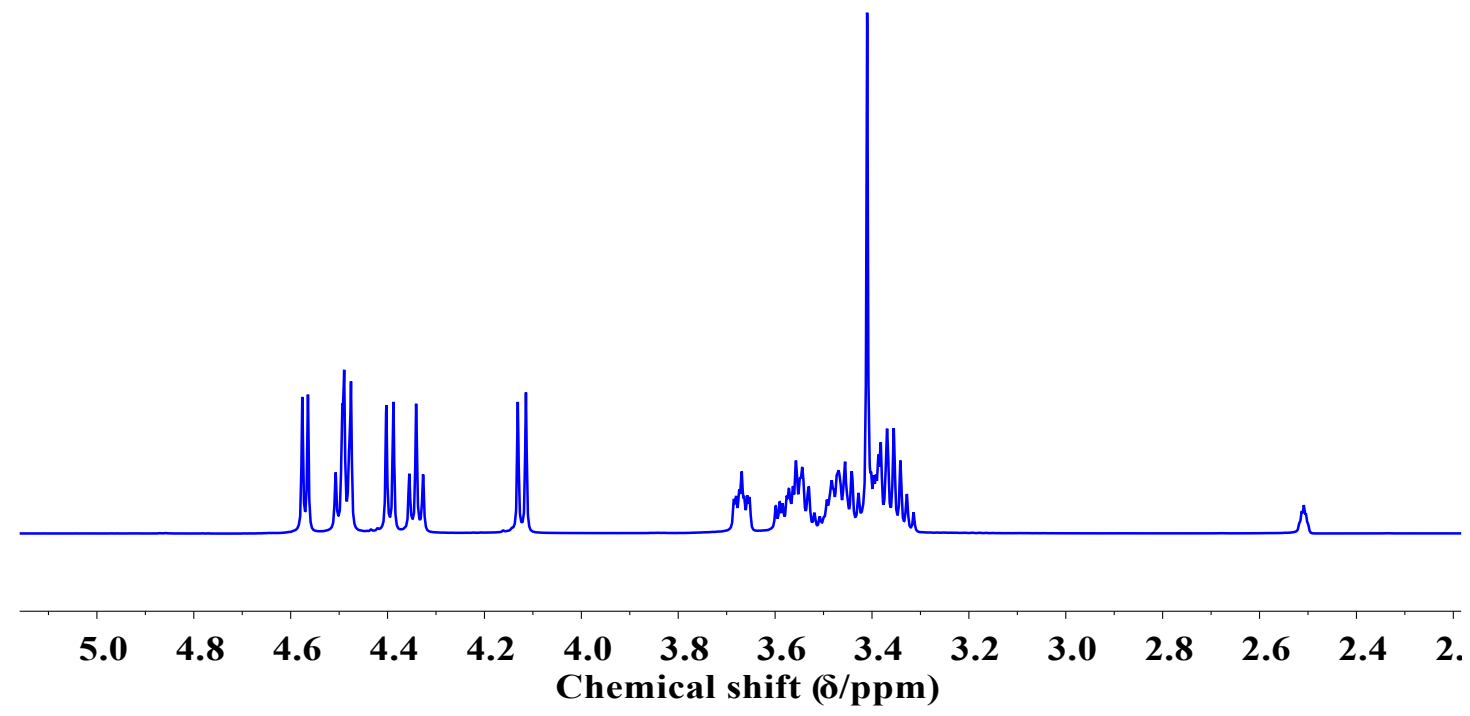

Figure S5. Conventional ${ }^{1} \mathrm{H}$ NMR spectrum of pure sorbitol in DMSO- $d_{6}$ at $298 \mathrm{~K}$. 


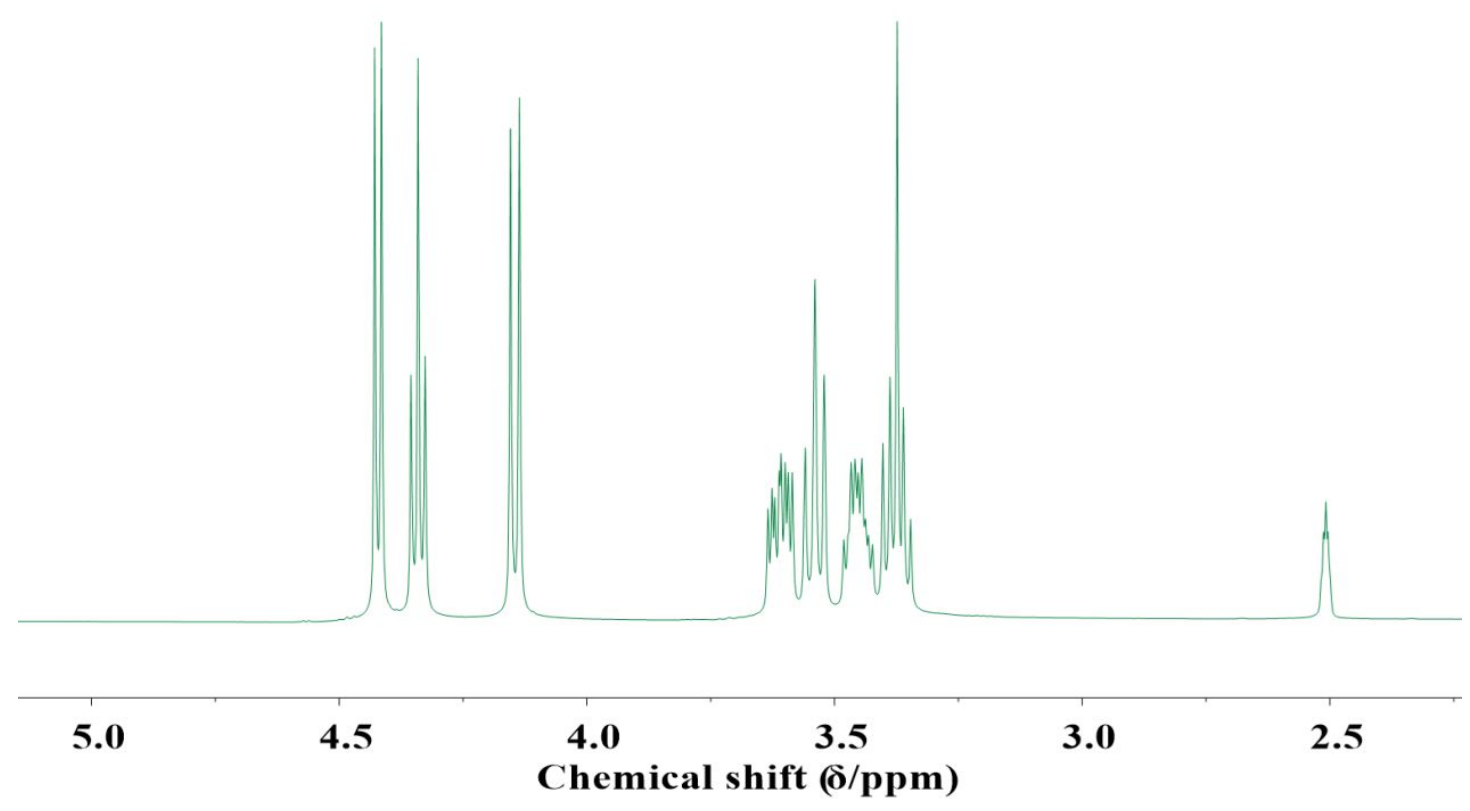

Figure S6. Conventional ${ }^{1} \mathrm{H}$ NMR spectrum of pure mannitol in DMSO- $d_{6}$ at $298 \mathrm{~K}$.
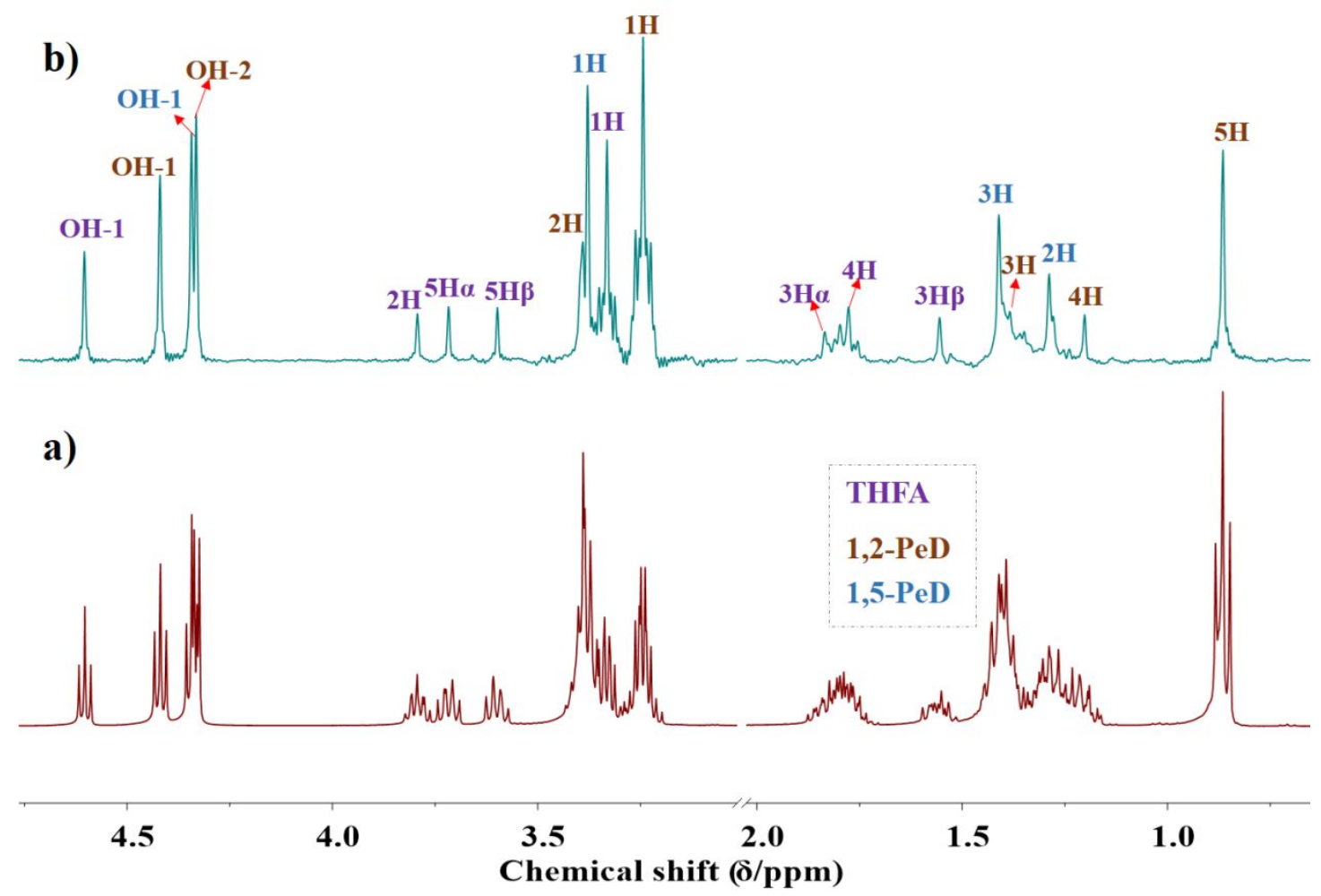

Figure S7. a) Conventional ${ }^{1} \mathrm{H}$ NMR spectrum b) 1D PSYCHE spectrum of sample 4 (THFA, 1,2-PeD and 1,5-PeD) in DMSO- $d_{6}$ at $298 \mathrm{~K}$. 

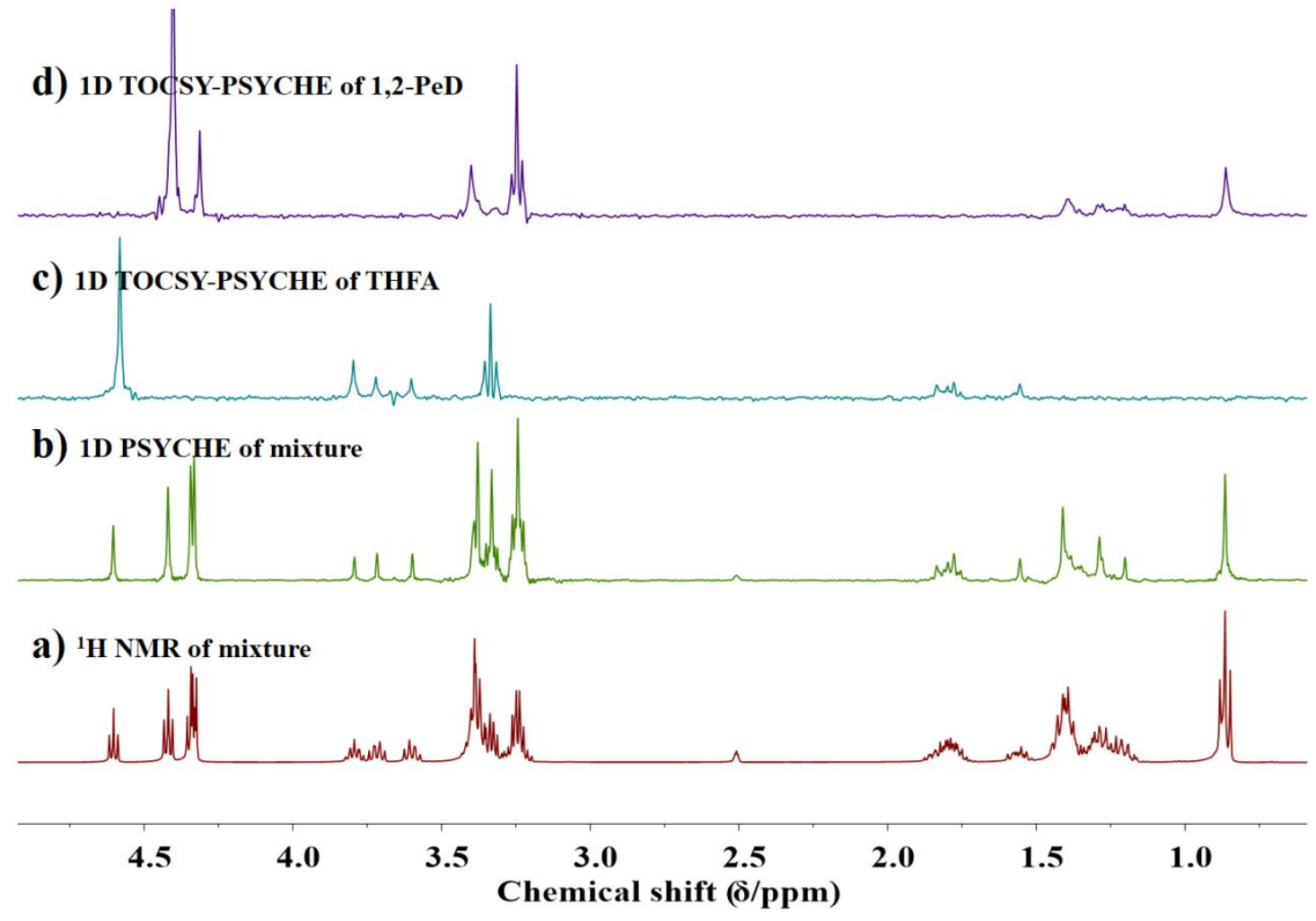

Figure S8. a) Conventional ${ }^{1} \mathrm{H}$ NMR spectrum of mixture (sample 4), b) 1D PSYCHE spectrum of mixture, c-d) 1D selective TOCSY-PSYCHE spectra (selective excitation frequencies at 4.60 and $4.42 \mathrm{ppm}$, respectively) of THFA and 1,2-PeD in DMSO- $d_{6}$ at $298 \mathrm{~K}$. 


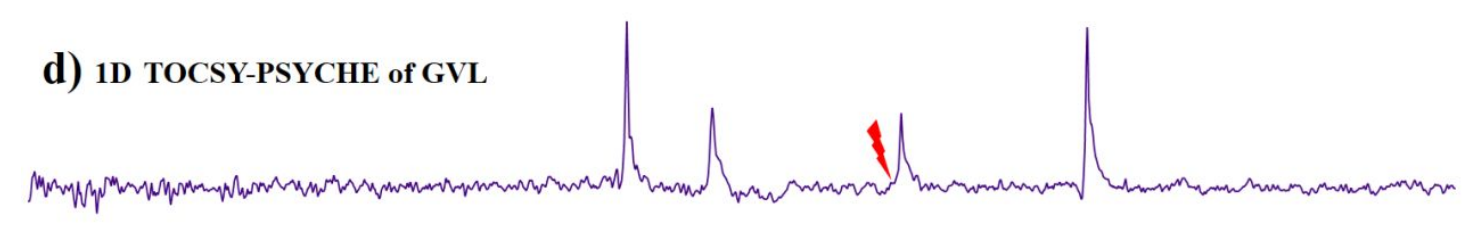

c) 1D TOCSY-PSYCHE of 4-hydroxyvaleric acid

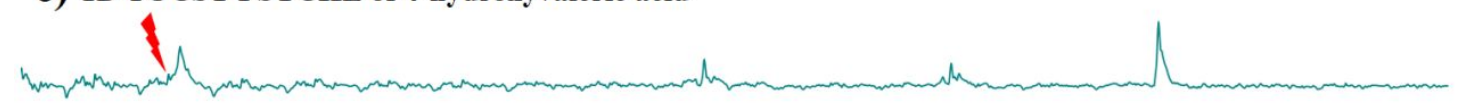

b) 1D TOCSY-PSYCHE of LA

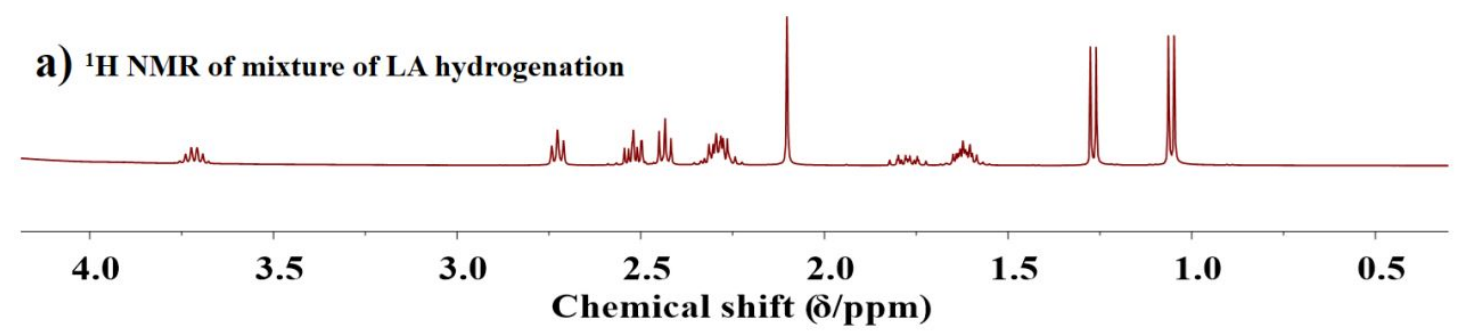

Figure S9. a) Conventional ${ }^{1} \mathrm{H}$ NMR spectrum of mixture (LA hydrogenolysis) and b-d) 1D selective TOCSY-PSYCHE spectra (selective excitation frequencies at 4.60 and $4.42 \mathrm{ppm}$, respectively) of LA, 4-hydroxyvaleric acid and GVL in $\mathrm{D}_{2} \mathrm{O}$ at $298 \mathrm{~K}$.

\section{Reference}

1. Dal Poggetto, G.; Castañar, L.; Morris, G. A.; Nilsson, M. A new tool for NMR analysis of complex systems: selective pure shift TOCSY. RSC Adv. 
2016, 6(102), 100063-100066. 